\title{
Tromboembolia pulmonar como complicación de COVID-19 en el puerperio. Reporte de un caso $=$
}

\author{
Pulmonary thromboembolism as a complication of CO- \\ VID-19 in the puerperium. Report of a case
}

Miguel Calva Maldonado ${ }^{1}$, Carlos Carrasco Vera² ${ }^{2}$ Noemí Judith García Contreras ${ }^{3}$

\begin{abstract}
December 31, 2019 an outbreak of pneumonia caused by a new coronavirus designated as SARS-CoV-2 was detected in the city of Wuhan (China). From a clinical point of view, it was found that patients can develop from a mild condition in the upper respiratory tract to severe pneumonia that are associated with respiratory distress progressing to severe respiratory failure. It has been described that the infection predisposes to hypercoagulability phenomena and venous thrombosis, recognizing pulmonary thromboembolism (PE) as the most frequent. Pregnancy is a physiological state with a high risk for the development of thromboembolic complications, increasing the risk factors for PTE during delivery and the puerperium. In the context of the association of SARSCoV-2 infection and pregnancy, there are few reports around the world as it is a recent pathology. In Mexico, according to official epidemiological records, COVID-19 has become the leading cause of maternal death, surpassing preclimactic events and obstetric hemorrhage, which until a few months ago were the leading cause. We present the case of a patient diagnosed with the new coronavirus who, during the middle puerperium, develops pulmonary thromboembolism, causing her death.
\end{abstract}

\section{Key words:}

Pulmonary thromboembolism, COVID-19, puerperium

Autor, profesor investigador tipo C asociado BUAP, titular de la especialidad en Anestesiología Hospital Universitario de Puebla, adscrito al Servicio de Anestesiología del Hospital General de Cholula. Puebla, México.

2 Coautor, jefe de Servicio de Anestesiología del Hospital General de Cholula. Puebla, México.

3 Colaborador, médico residente de tercer año de Anestesiología, Hospital General de Cholula. Puebla, México.

Fecha de ingreso: 02 de octubre de 2020

Fecha de aceptación: 22 de octubre de 2020

\section{ORCID}

https://orcid.org/0000-0002-3371-0987

\section{Correspondencia:}

Miguel Calva Maldonado

Benemérita Universidad Autónoma de Puebla Hospital General de Cholula.

drcalva55@gmail.com 


\section{RESUMEN}

El 31 de diciembre de 2019, se detectó en la ciudad de Wuhan (China) un brote de neumonía por un nuevo coronavirus designado como SARS-CoV-2. Desde el punto de vista clínico se encontró que los pacientes pueden desarrollar desde un cuadro leve de afección en vías respiratorias altas hasta cuadros de neumonía grave que se asocian a distrés respiratorio progresando a insuficiencia respiratoria grave. Se ha descrito que la infección predispone a fenómenos de hipercoagulabilidad y trombosis de localización venosa, reconociendo el tromboembolismo pulmonar (TEP) como el más frecuente. El embarazo es un estado fisiológico con alto riesgo para el desarrollo de complicaciones tromboembólicas, aumentando los factores de riesgo para TEP durante el parto y puerperio. En el contexto de la asociación de infección por SARS-CoV-2 y embarazo existen pocos informes alrededor del mundo al ser una patología reciente. En México, según registros epidemiológicos oficiales, el COVID-19 se ha convertido en la principal causa de muerte materna superando a los eventos preclámpticos y la hemorragia obstétrica, que hasta hace algunos meses, eran la primera causa. Se presenta el caso de una paciente diagnosticada con el nuevo coronavirus que, en el curso del puerperio mediato, desarrolla tromboembolia pulmonar causándole la muerte.

\section{Palabras clave:}

Tromboembolia

pulmonar,

COVID-19,

puerperio

\section{Introducción}

E 31 de diciembre de 2019, se detectó en la ciudad de Wuhan (China) un brote de neumonía por un nuevo coronavirus, designado como SARS-CoV-2 que en la actualidad presenta una distribución mundial[14]. Las mujeres embarazadas y sus fetos representan una población de alto riesgo durante los brotes de enfermedades infecciosas[12]. En México, hasta la última semana de septiembre de 2020 se han confirmado más de 710 mil casos positivos con 74.900 muertes, de las cuales 143 correspondieron a muertes maternas. Se ha descrito que este nuevo coronanavirus condiciona que los pacientes infectados desarrollen desde un cuadro leve de vías respiratorias altas hasta neumonía grave que se ha asociado a síndrome de distrés respiratorio que puede progresar a insuficiencia respiratoria grave y en muchos de los casos al fallecimiento[9],[10]. Se ha descrito de forma amplia los fenómenos de tipo trombóticos de localización venosa que pueden desarrollar estos pacientes, como el tromboembolismo pulmonar. La fisiopatología que relaciona el TEP con la infección por SARS-CoV-2 no se conoce bien y parece estar relacionada con un estado de hipercoagulabilidad[9],[10]. Estudios recientes han demostrado que niveles de dímero $D$ superiores a $1.000 \mathrm{ng} / \mathrm{ml}$ en pacientes con COVID-19 constituyen un índice pronóstico relevante de mortalidad. La respuesta inflamatoria sistémica y el daño endotelial asociados a la infección vírica activarían la coagulación, con aumento de la generación de trombina y disminución de los anticoagulantes naturales del organismo. La replicación viral desencadena la activación del complemento, con formación de C3a y C5a, capaces de reclutar neutrófilos, macrófagos, linfocitos y monocitos, responsables a su vez de la liberación masiva de citoquinas proinflamatorias (IL-1, IL-6, IL-8 e interferón $\gamma$ ) que favorecen la expresión del factor tisular (FT) de trombomodulina y de moléculas de adhesión endotelial activando la fibrinólisis[9],[13].

\section{Caso clínico}

Se expone el caso de una paciente con infección por COVID-19 que desarrolla tromboembolismo pulmonar, ocurrido en una unidad de cuidados intensivos de un centro hospitalario de referencia de atención de COVID-19. Se trata de femenino de 39 años de edad, multigesta con embarazo de 36 semanas de gestación con diagnóstico confirmado por prueba PCR de SARS-COV-2. Se programa de urgencia para resolución del embarazo por vía abdominal mediante cesárea tipo Kerr. Refiere iniciar con sintomatología 12 días previos a ingreso hospitalario caracterizada por cefalea, astenia, rinorrea y fiebre no cuantificada, a nivel privado inician medicación con azitromi- 
cina, paracetamol, dexametasona (no especificando dosis). Persistiendo sintomatología, realizan prueba PCR resultando positiva, por lo que se agrega a tratamiento levofloxacino, ceftriaxona y apixabán de forma ambulatoria (sin especificar dosis). El día previo a internamiento cursa con sensación de dificultad respiratoria por lo que acude al servicio de urgencias respiratorias. A su valoración inicial con criterios de insuficiencia respiratoria moderada de acuerdo a criterios de Berlín, PaFl calculada de 150 mmHg, puntaje de 3 en escala IC-RDOS y bajo riesgo en escala de CURB-65. Por criterio de ginecoobstetricia se decide realizar interrupción del embarazo por taquicardia fetal. Se realizó valoración preanestésica otorgando los siguientes riesgos: ASA IV, riesgo tromboembólico muy alto por Caprini y clasificación SOFA de 4 puntos con riesgo de mortalidad de $20 \%$. Se realizó procedimiento quirúrgico bajo anestesia neuroaxial de tipo subaracnoideo utilizando aguja whitacre \#25 dosis única, con uso de bupivacaína hiperbárica 10 mg más fentanilo 25 mcg, obteniendo producto único vivo. Sin complicaciones trasquirúrgicas, balance hídrico neutro, manteniendo estabilidad hemodinámica del paciente y con apoyo de oxígeno suplementario a través de mascarilla reservorio con un aporte de 10 litros/minuto, egresa al área de cuidados intensivos por protocolo hospitalario. Durante sus primeras horas de estancia en unidad de cuidados críticos se inicia anticoagulación con enoxoparina (Clexane ${ }^{\circledR}$ ) 60 mgs subcutáneos cada 24 h, continuando con aporte de oxígeno a $10 \mathrm{l} / \mathrm{min}$ a través de mascarilla reservorio con lo que mantiene $\mathrm{PaFl}$ calculada de 200 $\mathrm{mmHg}$; sin embargo, $24 \mathrm{~h}$ posteriores a su ingreso, debuta con deterioro súbito del estado de alerta, cianosis, disminución de la saturación de oxígeno hasta $75 \%$, taquipnea, hipertensión arterial con TAM de $120 \mathrm{mmHg}$, taquicardia paroxística supraventricular, gasometría con hipercapnia $\left(\mathrm{PaCO}_{2} 45 \mathrm{mmHg}\right)$ decidiendo realizar intubación de secuencia rápida. Al considerar factores de riesgo para tromboembolia pulmonar se realiza ecocardiograma transesofágico a las $12 \mathrm{~h}$ de evento, sin denotar alteraciones funcionales ni cambios estructurales; en el ultrasonido doppler de extremidades inferiores se observa trombosis venosa subaguda profunda a nivel de vena tibial anterior izquierda, por lo que se aumenta dosificación de enoxoparina a 80 mgs cada 12 h. Paciente desde la intubación inicial se mantuvo en modalidad volumen control con un volumen minuto de ocho litros, PEEP entre 5 a $8 \mathrm{cmH}_{2} \mathrm{O}$, presión plateau $<30 \mathrm{cmH}_{2} \mathrm{O}$. Por la inestabilidad hemodinámica con la que cursó en las primeras $72 \mathrm{~h}$ requirió dosis altas de noradrenalina con lo cual mantuvo adecuadas metas perfusoras. Se realiza angiotac donde se identifica trombosis venosa masiva de vena cava inferior de tipo subaguda a nivel de la aurícula derecha hasta la vena femoral común con paso filiforme del medio de contraste (Figuras 1 y 2). El Servicio de Angiología por el alto riesgo de mortalidad decide no colocar

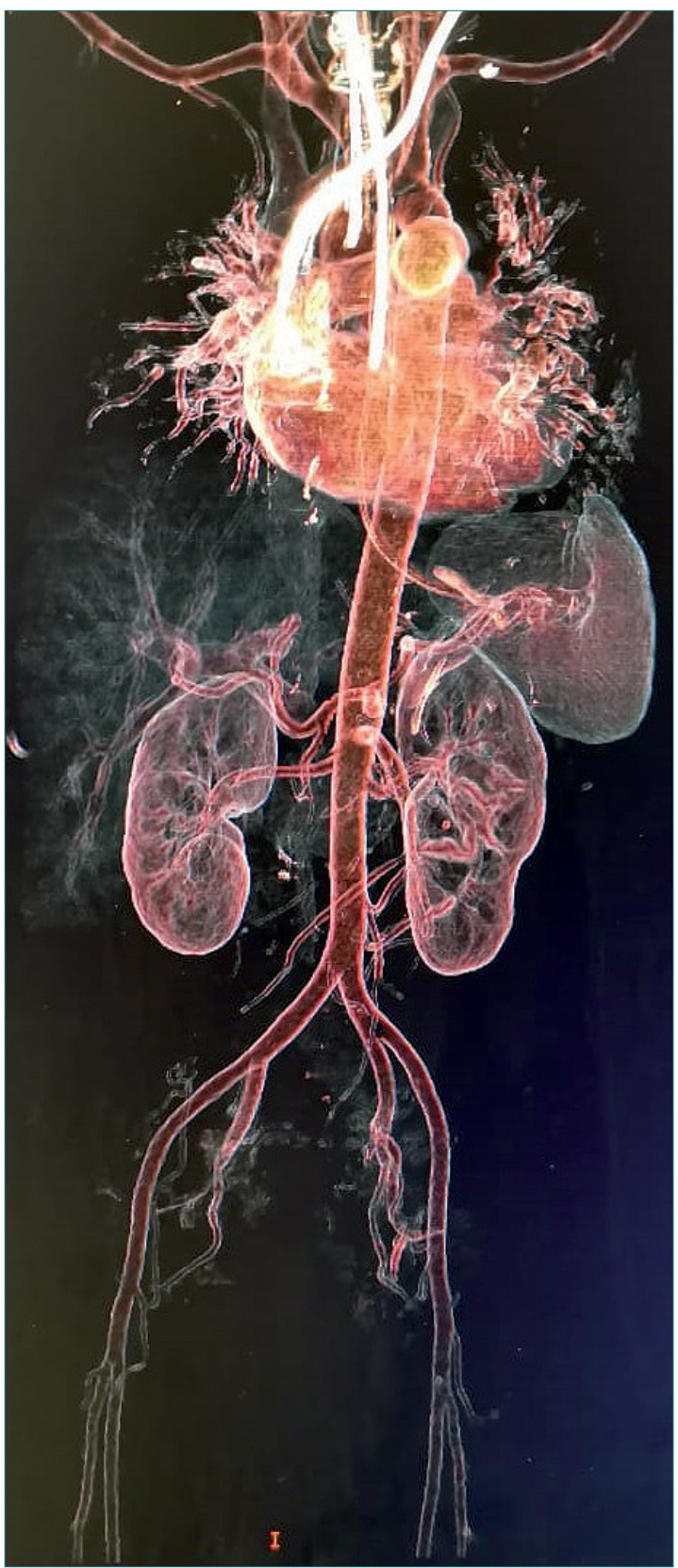

Figura 1. Angiotac realizada a la paciente. 


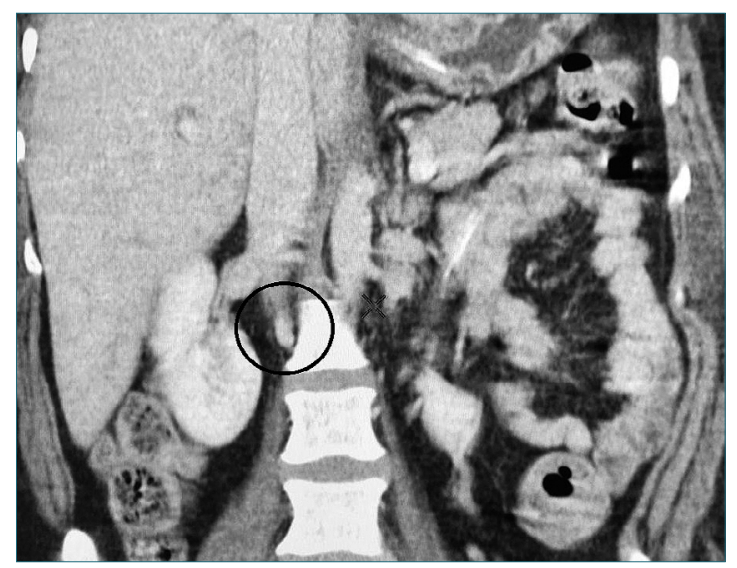

Figura 2. Se identifica parte del trombo de vena cava inferior.

filtro de vena cava y se opta por utilizar en sala de fluoroscopia trombólisis dirigida por catéter acelerada mediante ultrasonido EkoSonic (EKOS; Bothell, Washington, Estados Unidos); durante cateterismo y exploración no se identifica presencia de trombos, por lo que no se efectúa trombólisis. Paciente que durante los siguientes 12 días se mantiene en cuidados intensivos con persistencia de apoyo ventilatorio con requerimientos de $\mathrm{FiO}_{2}$ al 100\%, PEEP 8 - $12 \mathrm{cmH}_{2} \mathrm{O}$, presiones plateau elevadas y gasométricamente con tendencia a la hipercapnia, requiriendo constantemente el apoyo de noradrenalina a dosis elevadas y con marcadores inflamatorios elevados persistentemente. Paciente presenta de forma súbita TAM $<40 \mathrm{mmHg}, \mathrm{SPO} 2<50 \%$, cianosis generaliza$\mathrm{da}$, taquicardia sinusal que evoluciona a bradicardia con posterior asistolia; se presenta ausencia de pulso carotídeo y se registra trazo isoeléctrico, sin respuesta a reanimación cardiocerebropulmonar avanzada; se declara muerte tras 18 días de estancia en unidad de cuidados intensivos.

\section{Discusión}

La incidencia para tromboembolia pulmonar (TEP) en embarazadas es de cuatro a cinco veces mayor comparada con las mujeres no embarazadas[1]. En México, la incidencia es de 4,7 casos por cada 10.000 , con una mortalidad de 6,6\%[2]. La estasis venosa se presenta al final del primer trimestre del embarazo y se mantiene hasta alcanzar un punto máximo a las 36-38 semanas de gestación, agravado por el daño endotelial en los vasos pélvicos secundario a la compresión de la vena cava inferior y las venas ilíacas por el útero grávido, reduciendo la velocidad del flujo venoso hasta en 50\%[1],[2],[3]. El embarazo se caracteriza por cambios en el sistema inmunológico y fisiología pulmonar, con mayor susceptibilidad a infecciones virales y complicaciones más graves[11]. El diagnóstico de TEP en una mujer gestante empieza con la sospecha clínica, aunque el diagnóstico clínico carece de precisión y especificidad. Los signos y síntomas más frecuentes son: disnea (100\%), dolor torácico $(63,3 \%)$, taquicardia $(93,3 \%)$ y taquipnea $(93,3 \%)$ y sólo el 3\% son asintomáticas. Los gases sanguíneos demuestran alcalosis respiratoria, hipoxemia moderada y gradiente alvéolo-arterial de oxígeno mayor a $20 \mathrm{mmHg}$. El electrocardiograma es anormal hasta en el $90 \%$ de las mujeres embarazadas con TEP; lo más observado es la taquicardia sinusal, aunque puede presentarse inversión de la onda $T$, depresión del segmento ST y bloqueo de rama derecha del Haz de His. El ecocardiograma es un método no invasivo que identifica de forma indirecta la gravedad de la obstrucción vascular a través del grado de hipertensión arterial pulmonar y diferentes estadios de disfunción del ventrículo derecho[5].

La fisiopatología que relaciona la TEP con la infección por SARS-CoV-2 no se conoce del todo y parece estar relacionada con un estado de hipercoagulabilidad[6],[10,[13]. La respuesta inflamatoria sistémica y el daño endotelial asociados a la infección vírica condicionan un aumento en la generación de trombina con disminución de los anticoagulantes naturales del organismo. El SARS-CoV-2 infecta las células endoteliales que expresan ACE2 (enzima convertidora de angiotensina II). Este daño endotelial activa el factor tisular, que genera trombina a partir de protrombina por acción del factor $\mathrm{X}$ activado; las plaquetas circulantes activadas se agregan y proporcionan la superficie fosfolipídica adecuada para la adhesión de los diferentes compuestos de la cascada de la coagulación así como activación del complemento, con formación de C3a y C5a, capaces de reclutar neutrófilos, macrófagos, linfocitos y monocitos, responsables de la liberación masiva de citoquinas proinflamatorias (IL-1, IL-6, IL-8 e interferón $\gamma$ ) que favorecen la expresión del factor tisular, trombomodulina y moléculas de adhesión endotelial que activan la fibrinólisis[7],[8]. Estudios histopatológicos en pacientes han demostrado daño alveolar difuso con infiltración de células $T$ perivasculares, membranas celulares alteradas y características angiovasculares distintivas con presencia de trombosis y microangiopatía en los vasos pulmonares[9]. Todo lo anterior dificultando un diagnóstico 


\begin{tabular}{|c|c|c|c|c|}
\hline & $\begin{array}{c}\text { Ingreso } \\
\text { hospitalario }\end{array}$ & $\begin{array}{c}\text { Ingreso a cuidados } \\
\text { críticos }\end{array}$ & $\begin{array}{c}\text { Previos } \\
\text { a trombólisis }\end{array}$ & $\begin{array}{c}\text { Últimos } \\
\text { laboratorios }\end{array}$ \\
\hline Hemoglobina $(\mathrm{g} / \mathrm{dL})$ & 14 & 13,8 & 9,7 & 12,7 \\
\hline Hematocrito (\%) & 39,5 & 38 & 28,7 & 37,9 \\
\hline Leucocitos (10^3/uL) & 10,3 & 14,9 & 11,2 & 23,04 \\
\hline Neutrófilos (10^3/uL) & 14,8 & 12,9 & 8,3 & 20,0 \\
\hline Plaquetas (10A^/uL) & 304 & 300 & 262 & 65 \\
\hline Glucosa (mg/dl) & 92 & 78 & 51 & 110 \\
\hline Creatinina (mg/dl) & 0,6 & 0,5 & 0,5 & 0,5 \\
\hline Sodio (mmol/L) & 138 & 140 & 142 & 142 \\
\hline Potasio (mmol/L) & 4,8 & 4,3 & 3,5 & 3,2 \\
\hline Calcio (mg/dl) & 9,6 & 9,2 & 7,3 & 5,8 \\
\hline TGO (UI/L) & 24 & 29 & 25 & 77 \\
\hline TGP (UI/L) & 26 & 17 & 13 & 33 \\
\hline $\mathrm{FA}(\mathrm{UI} / \mathrm{L})$ & 177 & 141 & 128 & 144 \\
\hline $\mathrm{DHL}(\mathrm{UI} / \mathrm{L})$ & 321 & 407 & 420 & 450 \\
\hline $\mathrm{BT}(\mathrm{mg} / \mathrm{dl})$ & 0,4 & 0,5 & 0,4 & 0,8 \\
\hline TP (segundos) & 14,8 & 12,3 & 17,3 & 24,7 \\
\hline TTP (segundos) & 58,4 & 23,8 & 42,4 & 34,4 \\
\hline INR & 1,1 & 0,9 & 1,31 & 1,92 \\
\hline Fibrinógeno (mg/dL) & - & 997,9 & 638,8 & $1.071,6$ \\
\hline Dimero D (ng/ml) & 1.618 & - & 2.782 & 5.290 \\
\hline $\mathrm{PCR}(\mathrm{mg} / \mathrm{dL})$ & 222 & 210 & 124 & 141 \\
\hline $\mathrm{pH}$ & 7,39 & 7,07 & 7,41 & 7,24 \\
\hline $\mathrm{PCO}_{2}(\mathrm{mmHg})$ & 37 & 83 & 61 & 74 \\
\hline $\mathrm{PO}_{2}(\mathrm{mmHg})$ & 135 & 59 & 108 & 31 \\
\hline
\end{tabular}

TGO: Aspartato aminotransferasa; TGP: Alanino aminotransferasa; FA: Fosfatasa alcalina; BT: Bilirrubina total; DHL: Deshidrogenasa láctica. TP: Tiempo de protrombina; TTP: Tiempo de tromboplastina parcial; INR: Índice Internacional Normalizado; $\mathrm{PO}_{2}$ : Presión parcial de oxígeno; $\mathrm{PCO}_{2}$ : Presión parcial de bióxido de carbono PCR: Proteína $\mathrm{C}$ reactiva.

de TEP por la similitud en la sintomatología de COVID-19 en un paciente que ya presenta insuficiencia respiratoria[4]. Damos a conocer este caso clínico, ya que el manejo de pacientes obstétricas con infección por COVID-19, constituye un reto por la serie de complicaciones que pueden presentar.

\section{Referencias}

1. Navas A, Alba E. Pérez. Tromboembolismo pulmonar en el embarazo y puerperio: una patología mortal. Med Interna (Caracas). 2017;33(4):208-14.
2. Rojas-Sánchez Antonio et.al. Tromboembolia pulmonar en el embarazo y puerperio. Neumol Cir Torax. Vol. 73 - Núm. 1:4248. Enero-marzo 2014.

3. Ferrer OE. Trombosis venosa en el embarazo. Rev Med Clin Las
Condes. 2014;25(6):1004-18. https://doi.org/10.1016/S07168640(14)70650-9.

4. Departamento de Hemostasia y Trombosis. Instituto de Investigaciones Hematológicas Mariano R. Castex. Tromboembolismo 
venoso en el embarazo: desafíos en el diagnóstico. Hematología. Volumen 20 - Número Extraordinario. XII Congreso del Grupo CAHT: 238-242. Septiembre 2016.

5. Limberth Machado Villarroel,1 Diamanti Abraham Dimakis Ramírez2. Enfoque diagnóstico de la tromboembolia pulmonar. Acta Médica grupo Ángeles. Volumen 15, No. 1, enero-marzo 2017.

6. Middeldorp S, Coppens M, van Haaps TF, Foppen M, Vlaar $A P$, Müller $M C$, et al. Incidence of venous thromboembolism in hospitalized patients with COVID-19. J Thromb Haemost. 2020 Aug;18(8):1995-2002. https://doi.org/10.1111/ jth.14888 PMID:32369666

7. Franco-Moreno $A$, Muñoz-Rivas $N$, Mestre-Gómez B, TorresMacho J. Tromboembolismo pulmonar y COVID-19: un cambio de paradigma. Revista clínica española. 2020;220(7):459-61. https://doi.org/10.1016/j. rce.2020.05.006.
8. Bikdeli B, Madhavan MV, Jimenez D, Chuich T, Dreyfus I, Driggin E, et al.; Global COVID-19 Thrombosis Collaborative Group, Endorsed by the ISTH, NATF, ESVM, and the IUA, Supported by the ESC Working Group on Pulmonary Circulation and Right Ventricular Function. COVID-19 and Thrombotic or Thromboembolic Disease: Implications for Prevention, Antithrombotic Therapy, and Follow-Up: JACC State-of-the-Art Review. J Am Coll Cardiol. 2020 Jun;75(23):2950-73. https://doi. org/10.1016/j.jacc.2020.04.031 PMID:32311448

9. Ackermann M, Verleden SE, Kuehnel M, Haverich A, Welte $T$, Laenger $F$, et al. Pulmonary Vascular Endothelialitis, Thrombosis, and Angiogenesis in Covid-19. N Engl J Med. 2020 Jul;383(2):120-8. https://doi. org/10.1056/NEJMoa2015432 PMID:32437596

10. Dolhnikoff M, Duarte-Neto AN, de Almeida Monteiro RA, Ferraz da Silva LF, Pierre de Oliveira E,
Nascimento Saldiva $\mathrm{PH}$, et al. Evidencia patológica de fenómenos trombóticos pulmonares en COVID-19 severo. J Thromb Haemost. 2020. https://doi. org/10.1111/jth.14844.

11. Forestieri S, Marcialis MA, Migliore $\mathrm{L}$, et al. Relación entre embarazo y coronavirus: lo que sabemos. J Matern Fetal Neonatal Med. 2020;4:1-12. https:// doi.org/10.1080/14767058.2020 .1771692 PMID:32498581

12. Dashraath $P$, Jing Lin Jeslyn W, Mei Xian Karen L, et al. Enfermedad por coronavirus 2019 (COVID-19) pandemia y embarazo. Soy J Obs Gynecol. 2020;(20):30343-4

13. di Renzo GC, Giardina I. Enfermedad de Coronavirus 2019 en el embarazo: consideran trastornos tromboembólicos y la trombosis. Soy J Obstet Gynecol. 2020;22(20):30465-8.

14. Organización Mundial de la Salud. Informe de la misión conjunta OMS-China sobre la enfermedad por coronavirus 2019 (COVID-19). Genebra; 2020. 